Decision Making in Manufacturing and Services

Vol. 13 • $2019 \bullet$ No. 1-2 • PP. 5-15

\title{
A SPC Strategy for Decision Making in Manufacturing Processes
}

\author{
Alain Gil Del Val*, Bartosz Sawik**, Alba Agustín***, \\ Javier Faulín***, Pedro María Diéguez****
}

Abstract. Tapping is an extensively employed manufacturing process by which a multi-teeth tool, known as a tap, cuts a mating thread when driven into a hole. When taps are new or slightly worn, the process is under control and the geometry of the resulting threads on the workpiece is correct. But as the tap wear increases, the thread geometry deviates progressively from the correct one and eventually the screw threads become unacceptable. The aim of this paper is to outline the development of a statistical process control strategy for decision making based on data coming from the current signal of the tap spindle for assessing thread quality. It could operate on line, and indicates when the tap wear is so critical that, if the process were continued, it would result in unacceptable screw threads. The system would be very cost-effective since the tapping process could be run without any operator intervention.

Keywords: tapping, SPC, decision, quality, PCA

Mathematics Subject Classification: 90B50, 93C95, 28A12

Submitted: May 16, 2018

Revised: July 22, 2019

(C) 2019 Authors. This is an open access publication, which can be used, distributed and reproduced in any medium according to the Creative Commons CC-BY 4.0 License. License requiring that the original work has been properly cited.

\section{INTRODUCTION}

Tapping by cutting is one of the most common operations in manufacturing. It consists of cutting internal threads on the wall of a previously drilled hole by means of a tool called a tap that has cutting edges on its chamfered periphery. Tapping is a difficult operation due to the large number of cutting edges involved and the complicated synchronization necessary between the rotational and the feed movements of the tap, a task particularly difficult at high speeds (Gil Del Val et al., 2013; Gil Del Val et al., 2015).

* Tecnalia Research and Innovation, Donostia - San Sebastián, Gipuzkoa, Spain, e-mail: agildelval@hotmail.com

** AGH University of Science and Technology, Faculty of Management, Department of Applied Computer Science, Krakow, Poland, e-mail: bsawik@zarz.agh.edu.pl

*** Public University of Navarre, Institute of Smart Cities, Integrated Group of Logistics and Transportation, Pamplona, Spain, e-mail: albamaria.agustin@unavarra.es, javier.faulin@unavarra.es

**** Public University of Navarre, Department of Engineering, Pamplona, Spain,

e-mail: pmde@unavarra.es 
In this paper, we detail the experimental development of Statistical Process Control (SPC) strategy for decision making aimed at predicting the point in time when the tapped threads become unacceptable as a result of tap wear.

Chen et al. (1990) worked on tap modeling, and has focused on fault detection and classification for diagnosis purposes. Liu et al. (1991) continued this work using a neural network to classify the faults. Both papers relied on intrusive sensors. Further work along these lines was conducted by Li et al. (2002), whose system detects the same three types of faults of the previous papers but used less intrusive sensors. Even so, the sensors utilized involve an additional cost, and for training purposes the system requires production of unacceptable threads for each of the types of faults the system is to detect. The main problem of these last two papers is that they use intrusive sensors to diagnose the faults in tapping operations. However, manufacturers prefer tapping operations without intrusive sensors.

Although Li et al. (2002) used non-intrusive sensors and this reduces costs, we present a more cost effective solution in which the electrical current signal is acquired directly from the I/O module of the CNC machine itself, and hence no sensors are required. In contrast with earlier research on tap modeling, our emphasis is on process monitoring of the tap tool and on the decision-making of the corresponding thread quality, rather than on fault detection and classification of the tapping process with respect to a specific set of process conditions. This approach is close to the necessity of manufacturers' requirements, but this strategy is complex to operate in real time in a manufacturing cell.

A sophisticated monitoring and diagnosis approach for tapping was presented by Liu et al. (2013) based on features of torque and forces (thrust and lateral) from a Kistler dynamometer chosen by sequential forward search. An adaptive neuro-fuzzy interface system was used to classify the same five abnormal tapping processes described in the previously cited papers. In contrast to this complex manipulation of the data, which might not be intuitive for an operator, our approach uses well-established techniques for determining linear combinations of parameters to monitor (i.e. PCA) together with a powerful detection method from the statistical process control literature that provides an easy to understand chart. In addition, our approach has the advantage of interpretability, as the two principal components monitored are averages of certain areas under the torque profile.

Zhou et al. (2016) have studied two modifications to the conventional PCA-based monitoring methods to cope with the non-normal distribution and time-varying nature of the process data. Two algorithms are tested on the real process data to verify their effectiveness in the early abnormality detection of iron-making process.

Currently, Oezkaya and Biermann (2018) have proposed the development of a geometrical torque prediction method to determine relative torque values for tapping processes with various tapping tools and diameters. The results have been validated with experimental tests. This research focuses on torque as a main signal to control and make decisions in tapping processes. Therefore, this paper selects torque as the main signal to provide decision making when the process is out of the tolerance range. Besides, Lorentz (1989) was the first to use the principal component analysis in tapping processes. The results were good at improving the cutting and operational conditions. 
On the other hand, Statistical Control Process (SPC) has been used to improve the quality of the product and avoid faults, which cause industrial costs to soar. Shu and $\mathrm{Wu}$ (2011) propose allocate control charts in a multistage process in order to detect industrial faults. These two studies validate the control charts with different examples: automotive assembly and a hood assembly, respectively. In addition to this, the optimization of inspection requires the methodologies to raise customer satisfaction. M. Oppermann et al. (2001) developed a quality cost model based on $\mathrm{SPC}$ in electronics production of big companies.

Finally, the diagnosis systems provided by data mining and machine learning are being implemented in the industry to control the quality of the product and to avoid faults. A fault is a high industrial cost owing to the time, material and energy. Kiluk (2017) proposed a diagnostic information algorithm based on machine learning strategies to identify the dynamic faults in a heating process. Kiluk (2014) developed an algorithm-based diagnostic knowledge has dynamic properties that can work as a predictor to evaluate the faults in a thermal process.

The aim of this paper is to develop a SPC strategy for decision making when the thread quality is close to being beyond tolerance range. The paper has the following structure: firstly, set up, signal and measurement results will be shown. Secondly, Principal Component Analysis will be conducted to reduce the number of parameters from the monitor signal. Thirdly, a decision making methodology will be developed to guarantee the quality in the mechanical operation. Finally, Generalized Variance (GV) control charts will illustrate the decision making to stop the tapping process before a lack of thread quality ensues.

\section{SET UP, SIGNAL \& EXPERIMENTAL RESULTS}

Figure 1 illustrates a CNC machining centre where the tapping operations are performed. Taps are coated with Titanium Nitride (TiN) and their metrics are M10 × $1.5 \mathrm{~mm}$, respectively.

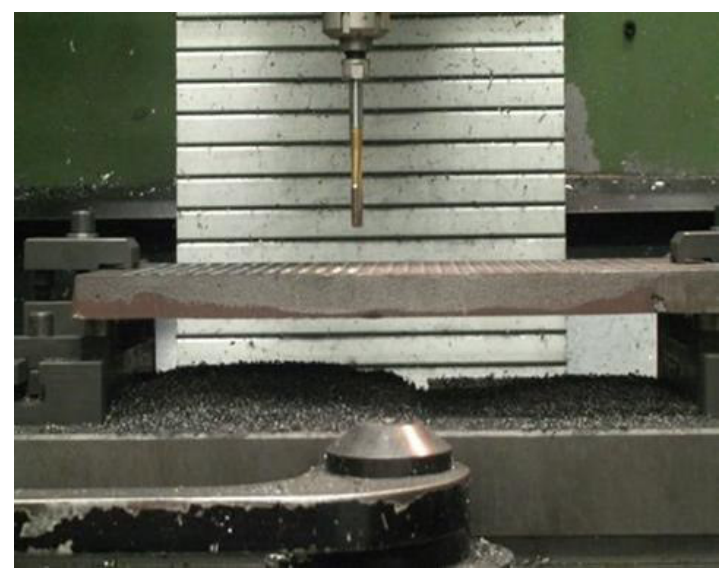

Fig. 1. CNC machining centre where the tapping operations 
Workpiece material is nodular cast iron (GGG50) in plates, whose dimensions are 250 by $450 \mathrm{~mm}$ and $20 \mathrm{~mm}$ thick. The cutting speed is $65 \mathrm{~m} / \mathrm{min}$ in dry conditions to avoid coolant. To assess thread quality, all tapped threads are inspected by a "go / non-go" gauge.

The electrical current from the I/O module of the CNC machine was selected to make a decision about the thread quality. Therefore, Figure 2a illustrates the torque sensitivity as acceptable and unacceptable thread profiles are superposed. The effect of wear on the tapped threads quality can be appreciated in Figure 2b. As a consequence, the go gauge cannot fit in the threaded hole.

a)

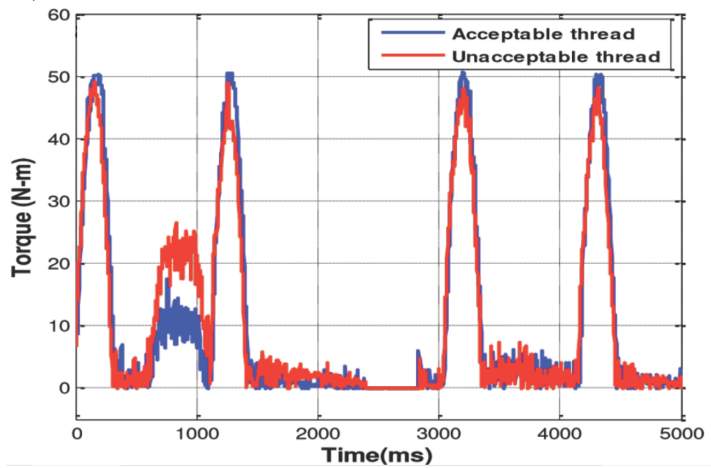

b)

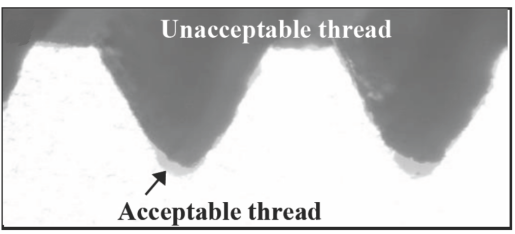

Fig. 2. The torque sensitivity as acceptable and unacceptable thread profiles: a) spindle motor torque signals from acceptable and unacceptable threads; b) lack of quality: non-defective and defective thread profiles

Six taps of the same characteristics have been tested and results are shown in Table 1. The second column indicates the number of tapped holes that passed the "go / non-go" gauge test. The process continued until an experienced operator declared that the tap end of life by catastrophic failure was close, and hence the tap was changed. Therefore, the third column indicates the total number of manufactured threads. The difference between two columns indicates the number of unacceptable threads.

Table 1. Number of tapped holes corresponding to the 6 HSSE M10 $\times 1.5$ taps tested

\begin{tabular}{|c|c|c|}
\hline Tap & Acceptable threads & Total threads \\
\hline 1 & 175 & 205 \\
\hline 2 & 108 & 120 \\
\hline 3 & 37 & 40 \\
\hline 4 & 60 & 80 \\
\hline 5 & 81 & 100 \\
\hline 6 & 109 & 120 \\
\hline
\end{tabular}


Parameters $A_{1}, A_{3}, A_{5}$ and $A_{7}$ represent the torque evolution area of the main spindle motor in the acceleration or deceleration periods in cutting and reverse stages as can be shown in Figure 3. Parameter $\mathrm{A}_{2}$ is the torque evolution area of the main spindle motor when the tap cuts threads. Parameter $\mathrm{A}_{4}$ is to keep synchronism between rotation and the feeding movements. Parameter $A_{6}$ is the tap torque evolution area during tap reverse.

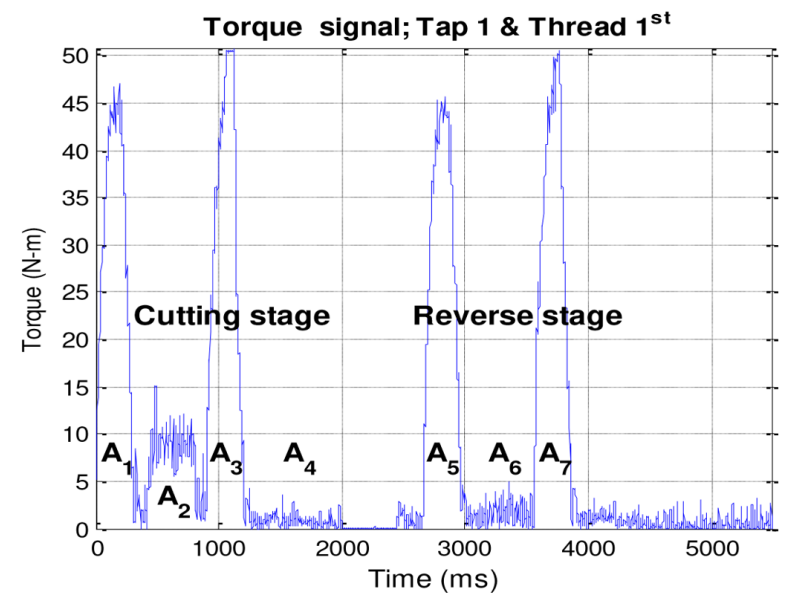

Fig. 3. The area parameters from current signal for decision making to assess the thread quality

With all these parameters, decision-making is complicated and consequently, the parameter number is reduced. For this task, the Principal Component Analysis (PCA) will be applied.

\section{PRINCIPAL COMPONENT ANALYSIS}

Principal Component Analysis (PCA) is a well-established statistical technique that expresses multivariate data as a set of linear functions of "latent" variables (the principal components, PCs).

Jackson (1991) was the first to propose the use of principal components for the quality monitoring of multivariate processes.

Let $\boldsymbol{X}$ be a $n \times p$ matrix containing the $n$ observations of the $p$ responses or signals of interest (in the tapping process, these are the $A_{i}$ torque area values, so $p=7$ ). It is good practice to standardize each column of $\boldsymbol{X}$ such that it has a mean of zero and a variance of 1 (hence, the values in the $\boldsymbol{Z}$ matrix can be negative or positive, contrary to the actual area measured which are obviously nonnegative):

$$
\begin{gathered}
Z=X-X S, \text { where }=\operatorname{diag}\left\{1 / S_{x^{i}}\right\} \\
s S=\operatorname{diag}\left\{1 / S_{x^{i}}\right\}
\end{gathered}
$$


and where $S_{x^{i}}$ is the standard deviation of the $i^{\text {th }}$ column of $\boldsymbol{X}$. The principal components $\boldsymbol{Y}$ are linear combinations of the (standardized) data:

$$
\boldsymbol{Y}=\boldsymbol{Z} \cdot \boldsymbol{V}
$$

where matrix $\boldsymbol{V}$ is found in such a way that the variance-covariance matrix of $\boldsymbol{Y}$ is diagonal (and hence, the PCs are orthogonal). We have that:

$$
\operatorname{cov}(\boldsymbol{Y})=\boldsymbol{V}^{\prime} \cdot(\boldsymbol{Z}) \cdot \boldsymbol{V}
$$

and therefore $\boldsymbol{V}$ is the matrix that diagonalizes $\operatorname{cov}(\boldsymbol{Z})$. This is well-known by Jackson (1991) to be achieved by the $p \times p$ matrix of eigenvectors of $\operatorname{cov}(\boldsymbol{Z})$ (the eigenvectors are orthonormalized, i.e., $\left.\boldsymbol{V}^{\prime} \boldsymbol{V}=\boldsymbol{I}\right)$. Thus, the PCs $\boldsymbol{Y}$ point in $p$ orthogonal directions in $p$-dimensional space where the data varies the most. The dimensionality of the problem is reduced by selecting only a subset of $k(k<p)$ PCs that explain most of the variance in $\boldsymbol{Z}$. The proportion of variability explained by the ith $\mathrm{PC}$ is given by $\lambda_{i} / \sum_{j=1}^{p} \lambda_{j}$ where $\left(\lambda_{1}, \lambda_{2}, \ldots, \lambda_{p}\right)$ are the eigenvalues of matrix $\operatorname{cov}(\boldsymbol{Z})$.

The PCA of the six taps tested, it can be concluded that the first two PCs, after simplification, are common to all taps, explain between $80 \%$ and $90 \%$ of the variability and are approximately equal to:

$$
\begin{gathered}
Y_{1}=0.25 \cdot\left(Z_{1}+Z_{3}+Z_{5}+Z_{7}\right) \\
Y_{2}=0.5 \cdot\left(Z_{2}+Z_{6}\right)
\end{gathered}
$$

\section{STATISTICAL PROCESS CONTROL}

SPC is an anticipatory technique which suggests stopping a process before the production of a defective part. Therefore, in the tapping process, the SPC scheme may suggest a decision to stop the process and replace the tap when the tap would have produced an additional number of acceptable threads until the end of tool life threshold. In decision making methods, this would constitute false positives (FPs). If the process were deemed in control past the end of life threshold of the tap this may not only result in defective threads but the tap may break inside the workpiece, which could result in considerable expense. In decision making analysis terms, these would correspond to false negatives (FNs), and would be therefore of more economic importance than false positives (FPs). As shown next, the proposed monitoring scheme provides a good trade-off between these costs, not giving false negatives and giving a reasonably low false positive rate.

Let $m$ be the number of observations (threads) in the training period. There are some studies that indicate how to choose $m$ in multivariate SPC, but they usually recommend a relatively large number of observations, at least 50 suggested by Montgomery (1996). If a too small training period is used, it is well-known by Montgomery (1996) that the statistical power of the control chart will be low. Based on our desire to obtain a monitoring scheme that works effectively for all tap behaviors observed in our experiments, the following empirical rule was used to determine the end of the 
learning period in each tap: the end of the training period should occur when the observed $\mathrm{A}_{2}$ parameter value equals 1.5 times the average $\mathrm{A}_{2}$ value observed during the five first tapped holes.

The GV chart requires the estimation of the instantaneous variance of the PCs, estimated following a suggestion in who use the moving ranges:

$$
V_{i}=Y_{i}+1-Y_{i}, i=1,2, \ldots, m-1
$$

and suggested as an estimator of the covariance matrix:

$$
S_{*}=\frac{1}{2 \cdot(m-1)} \sum_{i=1}^{m-1} V_{i} \cdot V_{i}^{\prime}
$$

The statistic plotted at time (tapped hole) $j$ on the Generalized Variance (GV) chart is the standard deviation of

$$
Z_{i j}=\frac{Y_{i j}-Y_{i m}}{\sqrt{S_{i *}}}, i=1,2, \ldots, p, \quad j=1,2, \ldots, p-1
$$

where $Y_{i m}$ is the average vector of PCs for each tap over the training period and $\mathrm{A}_{i}$. That is, the statistic plotted at time (or tapped hole number) $j$ on the GV chart is then

$$
G V_{j}=\frac{\sum_{i=1}^{p}\left(Z_{i j}-Z_{i m}\right)}{2}, j=1,2, \ldots, p-1
$$

The Upper Control Limit (UCL) of the GV chart is then calculated as

$$
U C L=B_{4} \cdot G \bar{V}
$$

where $G \bar{V}$ is average value of the GV statistic for each tap during the learning period, i.e.,

$$
G \bar{V}=\frac{1}{m} \cdot \sum_{i=1}^{m} G V_{i}
$$

and $B_{4}$ is a constant that depends on $m$ and corrects for bias Montgomery (1996). The process is deemed "out of control" when $G V_{j}>U C L$.

\section{DECISION MAKING METHODOLOGY}

Figure 4 illustrates the decision making methodology used to know the level of quality in the threads and the moment when the tapping process is out of control.

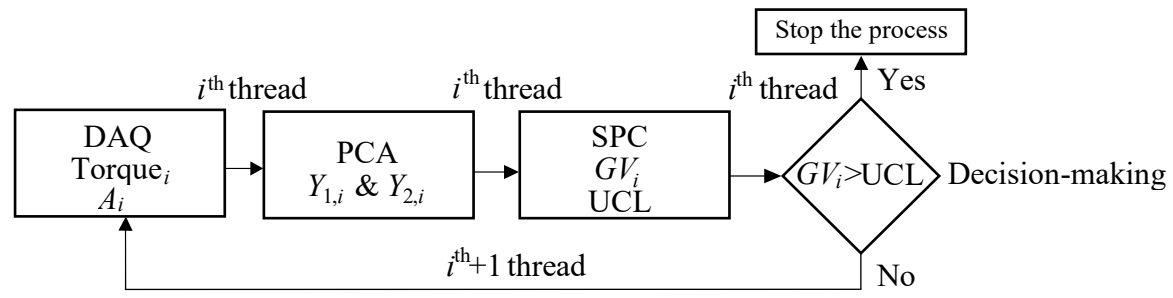

Fig. 4. Decision making methodology 
The data acquisition system registers the current signals. Then, several strategies were developed in Matlab ${ }^{\mathrm{TM}}$ to calculate the area parameters $\left(\mathrm{A}_{i}\right)$ from the torque signal, the principal components $\left(\mathrm{Y}_{1}\right.$ and $\left.\mathrm{Y}_{2}\right)$ and generalized variance values. For both statistical techniques, the learning period is when the observed $A_{2}$ parameter value equals 1.5 times the average $A_{2}$ value observed during the five first tapped holes. Once stablished the learning period, the following $i^{\text {th }}$ thread is sculpted and the method calculates the $\mathrm{A}_{i}$, principal components and $\mathrm{GV}$ value of $i^{\text {th }}$ thread. The last step of the methodology is decision making when the GV value of $i^{\text {th }}$ thread is compared with UCL value. If the GV value of $i^{\text {th }}$ is lower than the UCL value, the process continues with the following $i^{\text {th }}+1$ thread. However, if the GV value of $i^{\text {th }}$ is greater than the UCL value, the operator or machine can make a decision. On the one hand, the decision may be to stop the tapping process as it is out of control. The result is a high level of false positives (FP, threads that being in tolerances are considered unacceptable). On the other hand, the decision may be to continue until the real end tool life testing by the "go / non-go" gauge. In this case, false negatives (threads without being in tolerances are considered acceptable) might appear. This is a defective thread and this industrial cost is unsatisfactory. Therefore, this paper continues the tapping process in spite of the fact that the decision making is done as the tapping manufacturers want to know the rates of FPs and FNs, respectively.

\section{GV CONTROL CHARTS}

Taps 1, 3 and 6 represent reasonably well the behaviour of the whole set. For brevity's sake only these GV control charts (Figs. 5-7) are shown. In all figures, the blue line is the GV value, the horizontal red line is the UCL value, the vertical black line is end thread quality (ETQ, the real end of tool life) calculated by the "go / non-go" gauge and the red points are the FPs. There are no FNs in these charts.

Figure 5 illustrates the moment when the operator might make a decision to stop the process before mechanizing an unacceptable thread. This moment is in the $175^{\text {th }}$ thread. The GV control chart of tap 1 indicates that, from tapped hole number $175^{\text {th }}$ onwards, all threads are out of control (nonacceptable).

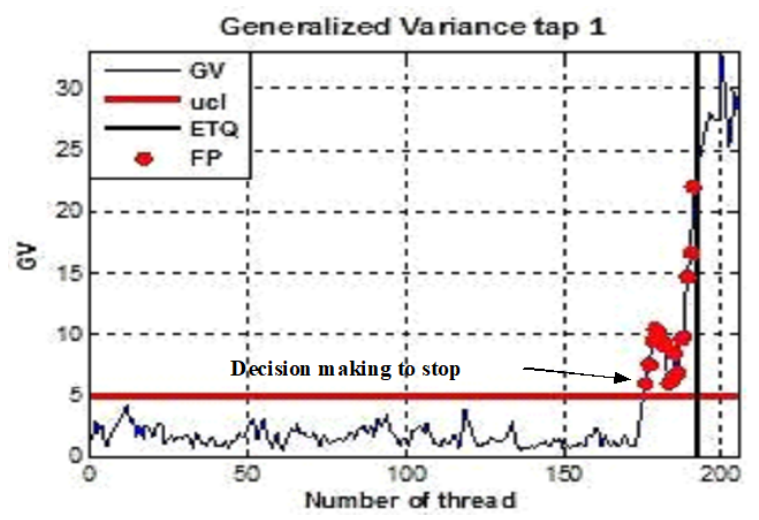

Fig. 5. SPC control chart for tap 1. First FP is the $175^{\text {th }}$ thread 
However, the tapping operation continued to know the tool life and the percentage of FPs as it has been described in the previous section. Experimental results have shown that the first non-acceptable one was actually number $192^{\text {nd }}$ as shown by the black vertical line. Therefore, the GV control chart for tap 1 provides an $8 \%$ FP. No FNs occured.

Figure 6 shows the tap 3 in spite of the fact that the GV behaviour is similar to tap 3. However, the great difference between two taps is the tool life as tap 3 is the shortest tool life. The decision making to stop could be on the $33^{\text {rd }}$ thread. It presents a $6 \%$ of $\mathrm{FP}$ and a $0 \% \mathrm{FN}$.

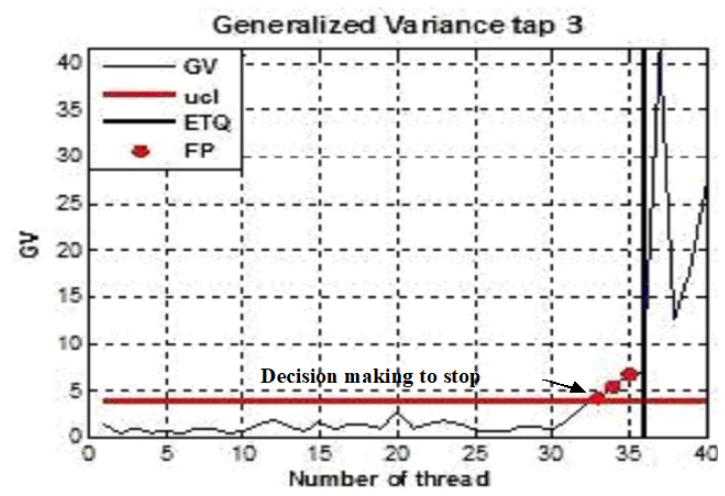

Fig. 6. $S P C$ control chart for tap 3. First FP is the $33^{\text {rd }}$ thread

Tap number $6 \mathrm{GV}$ control chart indicates that, again no FNs have occurred. The decision making to stop might be on $76^{\text {th }}$ thread which gives a $30 \%$ of FPs as it can be shown in Figure 7. This is a great tool cost, but there are no unacceptable threads, which are the greatest industrial costs.

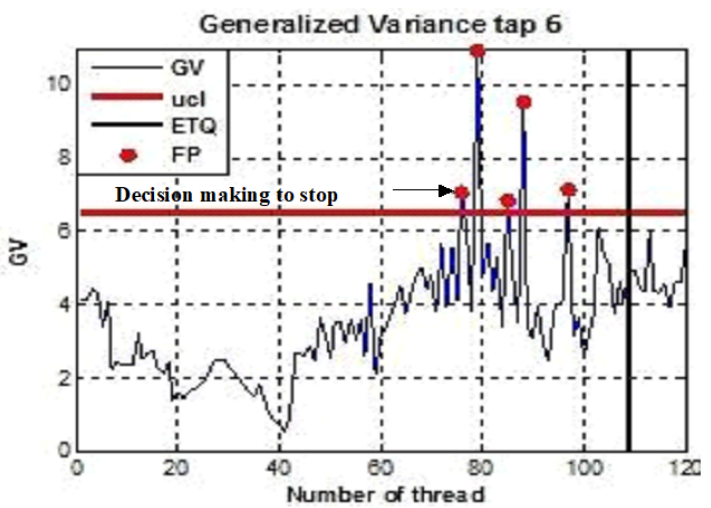

Fig. 7. SPC control chart for tap 6. First FP is the $78^{\text {th }}$ thread

The SPC strategy for decision making to stop the process before the lack of thread quality developed in this paper achieves the average FP rate across all the six 
taps in our experiments was close to $10 \%$ but no FNs were observed. In other words, the tapping process misses $10 \%$ of thread pieces but there are no defective pieces. A defective piece (i.e. a brake wheel) in which one thread is out of tolerances, has a high benefit and industrial cost in a manufacturing cell. Therefore, and considering that the cost of the tool varies between 10 and $30 \%$ of the total manufacturing cost, the cost penalty due to the early process stoppages represents only between 1.4 and $3.2 \%$. The managers considered these values acceptable for a manufacturing firm as a defect is an inadmissible industrial cost for them when the mechanized piece (e.g. a brake wheel) has all the added value. Besides, the comparison with the savings of having a monitoring system that allows working without an operator or with only partial operator service.

\section{CONCLUSIONS}

A SPS strategy for decision making to stop the process for assuring good quality of M10 $\times 1.5 \mathrm{~mm}$ threads machined with TiN Coated HSS taps and using torque signals of the spindle motor has been developed and validated for cast iron GGG50. It does not give any false negatives (a defective piece, which is an industrial cost) but it gives an average of $10 \%$ false positives, which increases tool costs. However, the cost penalty in most cases can be assumed.

The strategy detects when a particular tap goes out of control as a result of tool wear and it has enough generality to be applied to different types of work and tap materials with different cutting speeds, tap diameters and geometries.

The decision making methodology can work on line and can replace the operator vigilance task, while maintaining the thread quality at the same time.

\section{ACKNOWLEDGEMENTS}

This project, called IDI-20100674, was supported by the Centre for the Development of Industrial Technology answering to the Ministry of Economy, Industry and Competitiveness (Spanish Government). This research was also partly supported by AGH subsidy for maintenance and development of the research potential. The authors are grateful to anonymous reviewers for their comments.

\section{REFERENCES}

Chen, Y.B., Sha, J.L., Wu, S.M., 1990. Diagnosis of tapping process by information measure and probability voting approach. Journal of Engineering for Industry, 112, pp. 319-325.

Gil Del Val, A., Diéguez, P.M., Arizmendi, M., Estrems, M., 2015. Experimental study of tapping wear mechanisms on nodular cast iron. Procedia Engineering, 132, pp. 190-196.

Gil Del Val, A., Fernández, J., Arizmendi, M., Veiga, F., Urízar, J.L., Berriozábal, A., Axpe, A., Diéguez, P.M., 2013. On line diagnosis strategy of thread quality in tapping. Procedia Engineering, 63, pp. 208-217.

Jackson, J.E., 1991. A user's guide to principal components. John Wiley \& Sons, New York. 
Kiluk, S., 2014. Dynamic classification system in large-scale supervision of energy efficiency in buildings. Applied Energy, 132, pp. 1-14.

Kiluk, S., 2017. Diagnostic information system dynamics in the evaluation of machine learning algorithms for the supervision of energy efficiency of district heating-supplied buildings. Energy Conversion and Management, 150, pp. 904-913.

Li, W., Li D., Ni, J., 2002. Diagnosis of tapping process using spindle motor current. International Journal of Machine Tools \& Manufacture, 43, pp. 73-79.

Liu, T.-I, Lee, J., Liu, G., Wu, Z., 2013. Monitoring and diagnosis of the tapping process for product quality and automated manufacturing. The International Journal of Advanced Manufacturing Technology, 64(5-8), pp. 1273-1282.

Liu, T.I., Ko, E.J., Sha, S.L., 1991. Diagnosis of Tapping Operations Using an AI Approach. Journal Materials Shaping Technology, 9(1), pp. 39-46.

Lorentz, G., 1989. Principal component analysis in technology. Annals of CIRP, 38(1), pp. 107-109.

Montgomery, D.C., 1996. Introduction to statistical quality control. 3rd edition, John Wiley \& Sons, New York.

Oezkaya, E., Biermann, D., 2018. Development of a geometrical torque prediction method (GTPM) to automatically determine the relative torque for different tapping tools and diameters. The International Journal of Advanced Manufacturing Technology, 97, pp. $1465-1479$.

Oppermann, M., Sauer, W., Wohlrabe, H., 2001. Optimization of inspection strategies by use of quality cost models and SPC. Proceedings - Electronic Components and Technology Conference (Cat. No.01EX492), IEEE Xplore, pp. 293-297. 10.1109/ISSE.2001.931086.

Shu, M.-H., Wu, H.-C., 2011. Fuzzy X and R control charts: Fuzzy dominance approach. Computers and Industrial Engineering, 61, pp. 676-685.

Zhou, B.,Ye, H., Zhang, H., Li, M., 2016. Process monitoring of iron-making process in a blast furnace with PCA-based methods. Control Engineering Practice, 47, pp. 1-14. 